\title{
Sensitivity of strains of Candida albicans to jaritin, haloprogin, clotrimazole and miconazole
}

\author{
L. LANGSADL \\ M.D.
}

\author{
Z. JEDLICKOVA \\ M.D.
}

\section{Research Institute for Pharmacy and Biochemistry, Postgraduate Medical and Pharmaceutical Institute, Prague, Czechoslovakia}

\section{Summary}

The antifungal activity of jaritin and haloprogin against Candida albicans is similar. In vitro they had less activity than clotrimazole and miconazole, but the activity was independent of inoculum size.

\section{Introduction}

Several highly effective antimycotics, such as miconazole, clotrimazole and haloprogin, are used in several countries for the control of mycotic infections. The Research Institute for Pharmacy and Biochemistry in Prague has developed a haloprogin analogue, jaritin. It is a 2-methylthio-5-(3-iodopropargyloxy) pyrimidine. In this paper the antifungal activities of the new preparation jaritin are compared with those of haloprogin, clotrimazole and miconazole.

\section{Materials and methods}

Pure substances of these antimycotics were used for testing. The tests were performed with strains of Candida albicans isolated from patients with vaginal candidiasis and with laboratory strains of other Candida spp. The minimal inhibition concentration (MIC) was determined in both liquid and solid agar media by the dilution method. Strains previously cultivated on Sabouraud's glucose agar medium were washed off with saline and diluted to optical density (OD) 0.05 , measured at $520 \mathrm{~nm}$ in a spectrophotometer (SPECOL Zeiss). The diluted cultures were used as inocula. When testing the effect of the inoculum size for antifungal activities of the antimycotics, cultures were diluted to OD 0.01 and 0.05 equalling $1.6 \times 10^{4} \mathrm{CFU}^{*} / \mathrm{ml}$ and $2 \times 10^{8} \mathrm{CFU} / \mathrm{ml}$ respectively. A standardized inoculum of $0.05 \mathrm{ml}$ was then added to each test tube. Agar plates were inoculated with a Steer's replicator. The test tubes and plates were incubated at $28^{\circ} \mathrm{C}$ for $48 \mathrm{hr}$. The MIC was defined as the lowest concentration of an antimycotic inhibiting the growth after incubation at $28^{\circ} \mathrm{C}$ for $48 \mathrm{hr}$. The effect of proteins upon antifungal activities was determined in the same media

*CFU, colony-forming units supplemented by $10 \%$ of inactivated normal bovine serum (BIOVETA Ivanovice, Czechoslovakia). The composition of the liquid medium was Neopeptone (Difco) $1 \mathrm{~g}+$ glucose $2 \mathrm{~g} / 1000 \mathrm{ml}$ of distilled water. The solid medium was prepared in the same way as liquid medium, but with the addition of $15 \mathrm{~g}$ of Oxoid agar no. 3. The data were statistically tested by nonparametric Wilcoxon matched pairs signedrank test at the significance level 0.05 .

TABLE 1. Antifungal activities of jaritin and haloprogin in liquid medium. Inoculum size $2 \times 10^{8} \mathrm{CFU} / \mathrm{ml}$

\begin{tabular}{|c|c|c|c|c|}
\hline \multirow[b]{3}{*}{ Candida spp. } & \multicolumn{4}{|c|}{$\operatorname{MIC}(\mu \mathrm{g} / \mathrm{ml})$} \\
\hline & \multicolumn{2}{|c|}{ Jaritin } & \multicolumn{2}{|c|}{ Haloprogin } \\
\hline & 1 & 2 & 1 & 2 \\
\hline C. albicans & $0 \cdot 7$ & $1 \cdot 5$ & $0 \cdot 7$ & $12 \cdot 5$ \\
\hline C. tropicalis & $0 \cdot 3$ & 0.7 & $0 \cdot 15$ & $1 \cdot 5$ \\
\hline C. krusei & 0.7 & $3 \cdot 1$ & $6 \cdot 2$ & $25 \cdot 0$ \\
\hline C. pseudotropicalis & $6 \cdot 2$ & $12 \cdot 5$ & $25 \cdot 0$ & $25 \cdot 0$ \\
\hline C. lipolytica & $0 \cdot 15$ & 0.7 & 0.03 & $12 \cdot 5$ \\
\hline C. utilis & 1.5 & $6 \cdot 2$ & $25 \cdot 0$ & $25 \cdot 0$ \\
\hline C. humicola & $6 \cdot 2$ & $12 \cdot 5$ & $25 \cdot 0$ & $25 \cdot 0$ \\
\hline C. parapsilosis & $12 \cdot 5$ & $12 \cdot 5$ & $12 \cdot 5$ & $25 \cdot 0$ \\
\hline C. pelliculosa & $0 \cdot 3$ & 0.7 & 0.03 & $6 \cdot 2$ \\
\hline C. catenulata & $1 \cdot 5$ & $3 \cdot 1$ & $0 \cdot 3$ & $25 \cdot 0$ \\
\hline C. zeylanoides & $1 \cdot 5$ & $3 \cdot 1$ & $1 \cdot 5$ & $25 \cdot 0$ \\
\hline C. reukauffi & $1 \cdot 5$ & $3 \cdot 1$ & $0 \cdot 7$ & $25 \cdot 0$ \\
\hline
\end{tabular}

1. Medium without bovine serum.

2. Medium with $10 \%$ of inactivated bovine serum.

\section{Results}

The antifungal effect of jaritin and haloprogin in liquid medium against 12 laboratory strains of Candida spp. is shown in Table 1. C. pelliculosa

TABLE 2. The effect of inoculum size on antifungal activity against Candida albicans in liquid medium. Results - MIC (mg/l)

\begin{tabular}{lcc}
\hline & \multicolumn{2}{c}{ Inoculum size $(\mathrm{CFU} / \mathrm{ml})$} \\
\cline { 2 - 3 } & $2 \times 10^{8}$ & $1.6 \times 10^{4}$ \\
\hline Miconazole & $0.39-12.5$ & $0.025-1.56$ \\
Clotrimazole & $0.39-12.5$ & $0.025-0.10$ \\
Jaritin & $0.7-3.1$ & $0.39-0.7$ \\
Haloprogin & $0.3-6.2$ & $0.39-1.5$ \\
\hline
\end{tabular}


was most sensitive to haloprogin, and $C$. pseudotropicalis, C. utilis and $C$. humicola the least sensitive. The MICs of the strains regarded as resistant to haloprogin were sensitive to jaritin. The addition of $10 \%$ bovine serum produced significant differences in the sensitivities to both jaritin and haloprogin. The activity of miconazole and clotrimazole against C. albicans was shown to be dependent on inoculum size (Table 2).

Antifungal activities of jaritin, haloprogin, clotrimazole and miconazole against freshly isolated strains from patients with vaginal candidiasis were also tested on solid agar medium. The inoculum size was $1.6 \times 10^{4} \mathrm{CFU} / \mathrm{ml}$. The range of $\mathrm{MICs}(\mathrm{mg} / \mathrm{l})$ for jaritin was $0 \cdot 1-1 \cdot 5$; for haloprogin, $0 \cdot 2-6 \cdot 2$; for clotrimazole, 0.01-0.7; for miconazole, 0.1-3.1. There were no statistically significant differences between MIC values of jaritin and haloprogin, but there were differences between MIC values of jaritin, clotrimazole and miconazole.

Jaritin in the concentration $0.78 \mathrm{mg} / \mathrm{l}$ inhibited $48.9 \%$ of the strains. As many as $96.9 \%$ of the strains were inhibited by $1.5 \mathrm{mg} / \mathrm{l}$ of haloprogin. The concentration of $0.78 \mathrm{mg} / \mathrm{l}$ of miconazole inhibited $93 \%$ of strains (Fig. 1).

\section{Conclusions}

The antifungal activities of jaritin and haloprogin against collection strains of Candida are approximately equal. When testing freshly isolated strains of $C$. albicans, the same activity was found. Antifungal activities of jaritin and haloprogin do not depend on the inoculum size. The antifungal activities of clotrimazole and miconazole depend on the size of inoculum. With high inoculum size the activity of jaritin is higher than the activity of miconazole. With low inoculum size, antifungal activities of clotrimazole and miconazole are higher than those of jaritin.

The activity of all antimycotics tested was inhibited by the presence of serum proteins in the medium. Using freshly isolated strains of $C$. albicans in the lower concentration of inoculum $\left(1.6 \times 10^{4} \mathrm{CFU} /\right.$ $\mathrm{ml}$ ), clotrimazole was the most effective agent; miconazole came next; jaritin and haloprogin followed. No statistical differences between jaritin and haloprogin in antifungal activity were found.

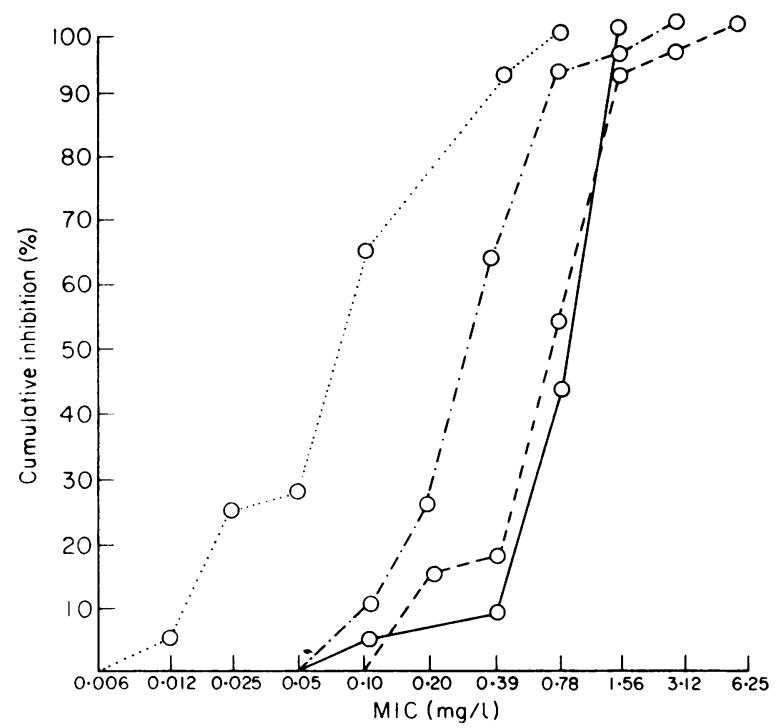

FIg. 1. Antifungal activity of antimycotics against fresh isolated strains of Candida albicans. Jaritin - ; haloprogin ---; miconazole--.--; clotrimazole.... .

\section{References}

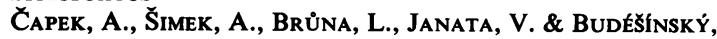
Z. (1972) [Antimicrobial agents. XV. Antimycotic effect of VUFB 9244, 2-methylthio-5-(3-iodopropargyloxy) pyrimidine.] Folia microbiologica, 17, 396.

Harrison, E.F., Zwadyk, P., BequetTe, R.J., Hanlow, E.E. \& TAVormina, P.A. (1972) Haloprogin: a topical antifungal agent. Applied Microbiology, 19, 746.
Van Cutsem, J.M. \& Thienpont, D. (1972) Miconazole, a broad-spectrum antimycotic agent with antibacterial activity. Chemotherapy, 17, 392.

WaitZ, J.A., Moss, E.L. \& Weinstein, M.J. (1971) Chemotherapeutic evaluation of clotrimazole/Bay b 5097, 1 (o-chloro- $\alpha$ - $\alpha$-diphenylbenzyl) imidazole. Applied Microbiology, 22, 891. 